American Journal of Immunology 1 (1): 24-30, 2005

ISSN 1553-619X

(C) 2005 Science Publications

\title{
The immunological aspects of Acanthamoeba infections
}

\author{
Naveed Ahmed Khan \\ School of Biological and Chemical Sciences, Birkbeck College, University of London \\ London, England, UK
}

\begin{abstract}
Acanthamoeba is a protozoan pathogen that is responsible for two recognized diseases; i) a blinding keratitis, most commonly associated with contact lens wear, and ii) a rare but fatal granulomatous encephalitis that usually is limited to immunocompromised patients. The fact that Acanthamoeba infections are generally limited to avascular cornea or immunocompromised patients suggests that the normal immune responses may be sufficient to control and/or eradicate these pathogens. Here we describe our current understanding of the immune responses to Acanthamoeba infections.
\end{abstract}

Key words: Acanthamoeba keratitis, Acanthamoeba granulomatous encephalitis (AGE), immune response

\section{INTRODUCTION}

Acanthamoeba spp. is a group of free-living amoebae that are widely distributed in the environment including, soil, air, water, pools etc. Based on their environmental distribution, Acanthamoeba can be considered as one of the most ubiquitous protozoa. It is not surprising that up to $100 \%$ of healthy individuals have been shown to possess anti-Acanthamoeba antibodies indicating our common exposure to these pathogens (1). Acanthamoeba were first discovered in 1930 as yeast (Cryptococcus) culture contaminants (2) and the genus Acanthamoeba was created (3). The term "amoebae" suggests amoeboid motion, i.e., crawling- like movement, common to other amoebae (Fig. 1). The term "acanth" (Greek: meaning spikes) was added to these amoebae due to the presence of spine-like structures (now known as acanthopodia) on the surface of these organisms (Fig. 2) as opposed to pseudopodia in other amoebae. The acanthopodia are most likely important in adhesion to surfaces, movement or capturing prey such as bacteria, algae, yeasts or small organic particles. Acanthamoeba feed by pinocytosis (a non-specific process associated with uptake of large volumes of solutes/particles by invagination of the plasma membrane) and/or phagocytosis (engulfing large particles which may require specific interactions) $(4-6)$.

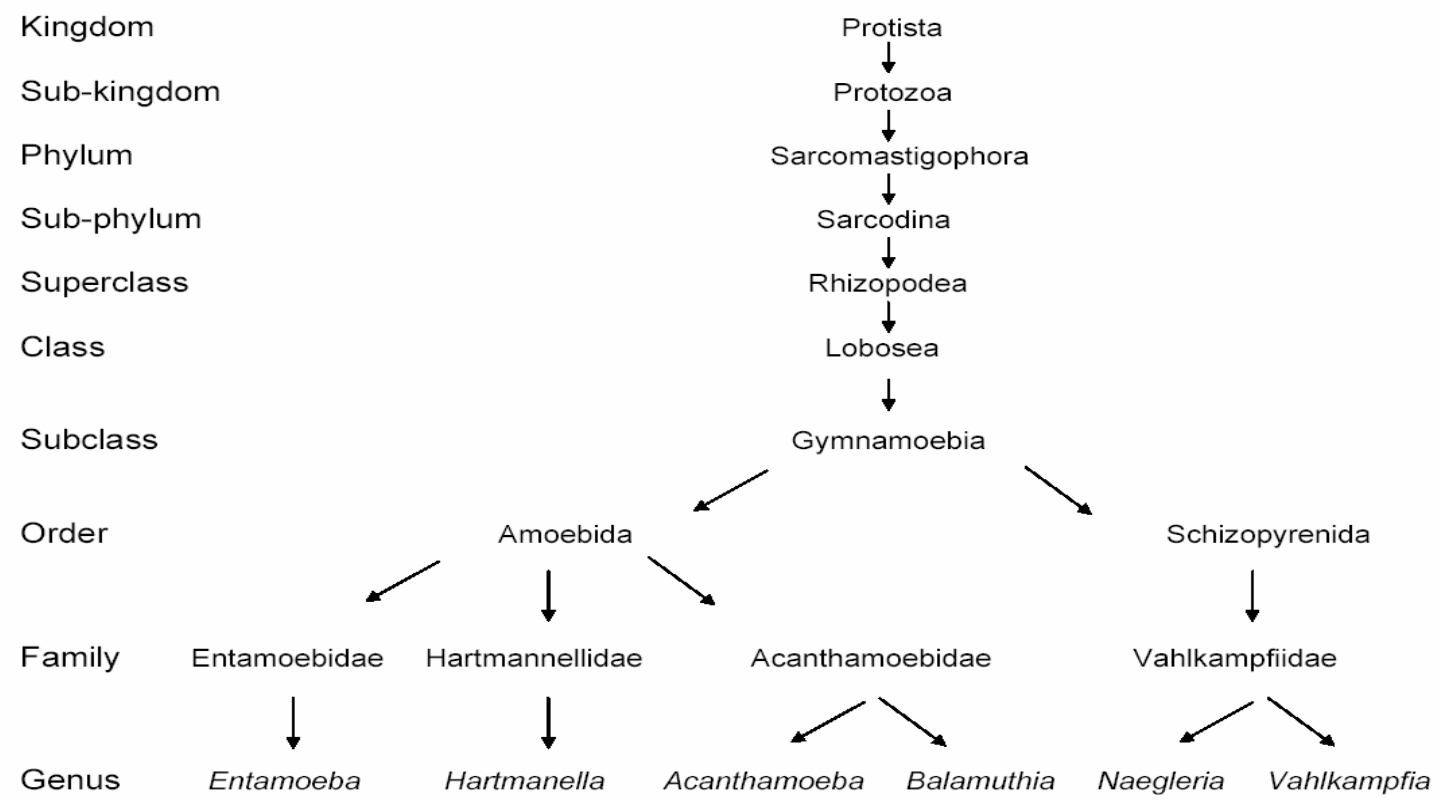

Fig. 1: The classification scheme of free-living amoebae. Modified from references 8 and 42 . 
The life cycle of Acanthamoeba consists of two stages, a vegetative trophozoite stage and a dormant cyst stage. The trophozoite possesses a single nucleus with a prominent nucleolus and divide by binary fission (i.e., parent cell mitotically divides into two daughter cells). However, under harsh conditions, such as lack of food, extremes in temperatures and $\mathrm{pH}$, Acanthamoeba transform into a non-dividing, double-walled resistant cyst form.

Genus Acanthamoeba consists of both invasive and non-invasive species. Pathogenic species of Acanthamoeba are frequently associated with keratitis, a painful eye infection that could result in loss of the vision and is typically associated with the use of contact lenses. Other infections due to pathogenic Acanthamoeba include skin ulcerations and a fatal granulomatous amoebic encephalitis (reviewed in 7-9). The first clearly identified Acanthamoeba granulomatous encephalitis (AGE) in humans was observed in 1972 (10) and the first Acanthamoeba keratitis case was reported 1974 (11). Since then, Acanthamoeba have become increasingly important microbes. This is partly due to increased numbers of immunocompromised patients and increased contact lens users.

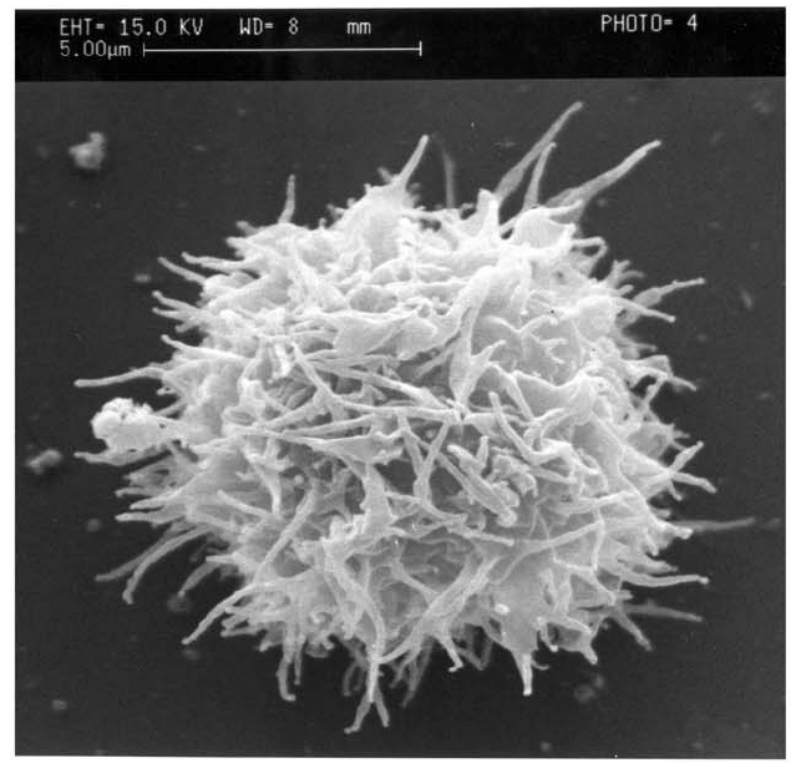

Fig. 2: Scanning electron micrograph of Acanthamoeba trophozoites exhibiting large number of acanthopodia (spine-like structures) on their surface.

\section{Acanthamoeba keratitis and the immune response The infection}

First reported in 1974 (11), Acanthamoeba keratitis has become a significant ocular infection. More than $85 \%$ Acanthamoeba keratitis cases are associated with the contact lens wear. The inappropriate cleaning/use of contact lenses together with the lack of personal hygiene are primary contributing factors in contracting Acanthamoeba keratitis. However in non-contact lens wearers, the presence of corneal trauma followed by exposure to contaminated water, soil, vector (biological or inert objects) is sufficient to produce Acanthamoeba keratitis $(12,13)$. The requirement of corneal trauma can be explained with the fact that the expression of Acanthamoeba-reactive glycoprotein(s) on damaged cornea is 1.8 times higher, than on intact corneas suggesting that corneal injury contributes to Acanthamoeba infection (14). Regardless of the status of the cornea, it is suggested that Acanthamoeba must be present in the trophozoite stage as cysts do not bind to the corneal epithelial cells (15). Once the corneal tissue is breached Acanthamoeba multiply to establish themselves in the host (incubation period may vary), followed by physically damaging the host tissue or by depriving it of nutrients and inducing excessive host immune response resulting in disease. The onset of symptoms could take from few days to several weeks. This is dependent on the inoculum size of Acanthamoeba and/or the extent of corneal trauma. Although symptoms may vary depending on the clinical management of the diseases but most commonly associated with tearing, epithelial defects, photophobia, redness, stromal infiltration, edema, stromal opacity together with excruciating pain due to radial neuritis, epithelial loss and stromal abscess formation with vision-threatening consequences. Other features may involve scattered sub-epithelial infiltrates, anterior uveitis, stromal perforation, the presence of scleral inflammation. Glaucoma is commonly reported and occasionally posterior segment signs such as nerve edema, optic atrophy and retinal detachment are observed. In untreated eyes, blindness may eventually result as the necrotic region spreads inwards (16).

\section{Host defenses}

Although avascular, cornea is protected by the host defenses provided by the eyelids and secreted tear film. The tear fluid produced by lacrimal system together with constant eye lid movement provides the first line of defense against Acanthamoeba keratitis. The tear film primarily contains lysozyme, lactoferrin, $\beta$-lysins, secretory immunoglobulin A (sIgA), prosglandins, and other compounds with antimicrobial and immunological properties $(17,18)$. Of the three distinct layers in the tear film, (i.e., the oil layer, the aqueous layer and the 
mucous layer), the aqueous layer is documented source of compounds with antimicrobial properties such as non-specific antimicrobial lysozyme, lactoferrin and specific sIgA. The role of $\operatorname{sIgA}$ in Acanthamoeba keratitis has been shown with the findings that i) Acanthamoeba keratitis patients show decreased levels of $\operatorname{sIg} \mathrm{A}$ and ii) $\operatorname{sg} \mathrm{A}$ (anti-amoebic) inhibit Acanthamoeba binding to corneal epithelial cells suggesting that sIgA plays an important role in the inhibition of amoebae binding to the corneal surface, thus preventing the infection (19-21). Overall, the tear film in conjunction with the blink reflex is highly effective in blocking microbial access to corneal epithelial cells and expelling amoebae from the surface of the eye and carries it to the conjunctiva. Although cornea is protected by only limited immune mechanisms, the conjunctiva is highly vascular with lymphoid tissue and contains mainly IgA-producing plasma cells, T-lymphocytes, natural killer cells and macrophages, which are highly effective in clearing Acanthamoeba and stimulating humoral and T-cell responses. For example, conjunctival macrophage depletion exacerbate Acanthamoeba keratitis symptoms in vivo (22). Thus in order to proceed with infection, Acanthamoeba must remain at the corneal surface and invade into the stromal tissue. Of interest, the tear film in contact lens wearers is shown to differ in terms of volume and make up and it seems that extended contact lens wear alters the levels of inflammatory mediators in tears and may contributes to increased inflammation (23). Other major changes due to contact lens wear include occlusion of atmospheric oxygen and thinning of the basal tear film, which is responsible for the metabolic compromise of the cornea and also leads to less resistance to microbial infections (24). Overall, this indicates the cornea is a stressed environment in the presence of contact lenses, and this affects many components of the tear film. This may in turn makes invasion by the amoebae more likely. In addition, the highly virulent strains of Acanthamoeba evade these primary defenses to traverse the cornea and invade into the stroma. Once in the stroma, Acanthamoeba secrete proteolytic enzymes causing stromal degradation leading to macrophages/neutrophil infiltration, which modulate B- and T-lymphocyte activity to clear Acanthamoeba. The macrophages provide defense against Acanthamoeba keratitis directly by clearing amoebae and by inducing an inflammatory response, in particular secretion of macrophage inflammatory protein 2 (MIP-2). This results in the recruitment of other immune cells such as neutrophils, which are highly potent in destroying Acanthamoeba in a myeloperoxidase-dependent manner $(25,26)$. Overall, these studies suggest that upon entry into the eye, Acanthamoeba has to deal with normal tear film containing the non-specific antimicrobial compounds together with the sweeping action of the eye lids as well as specific immunoglobulins and cell-mediated immunity (Fig. 3).

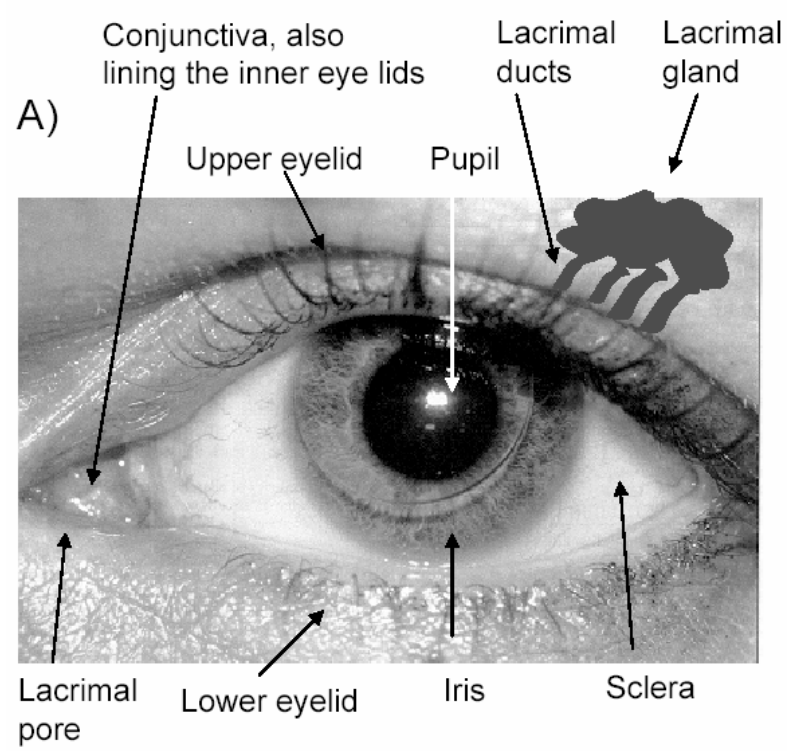

B)
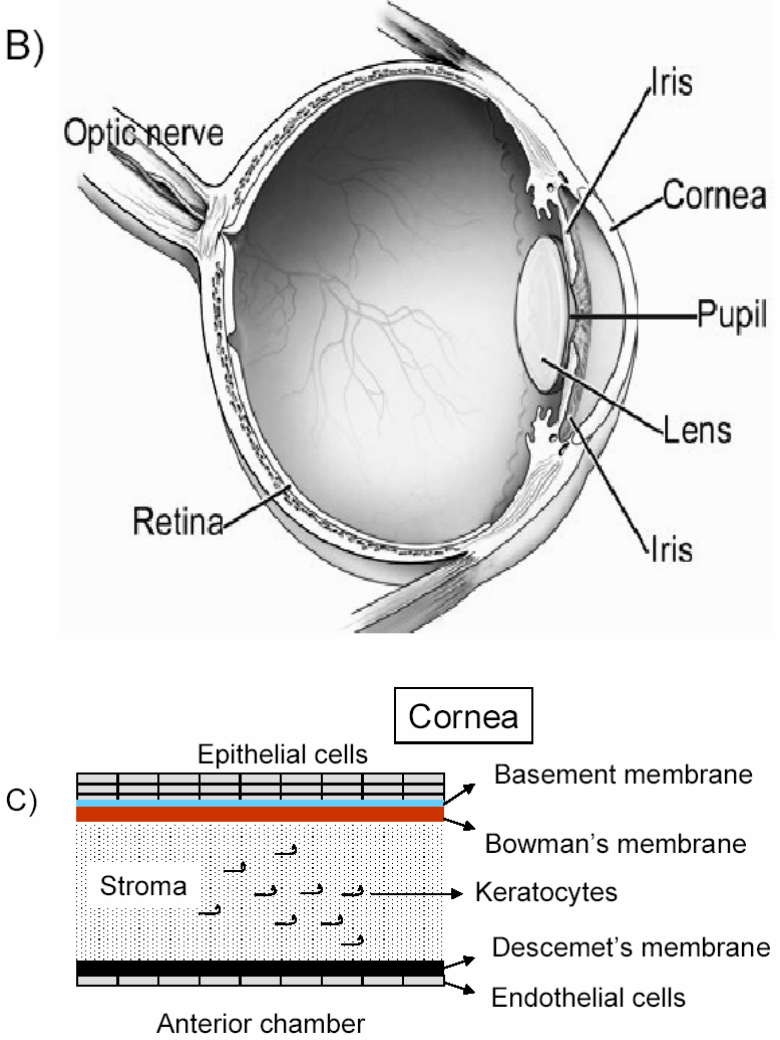

Fig. 3: The anatomy of the eye (A, B) and structure of the cornea $(\mathrm{C})$. 


\section{Acanthamoeba granulomatous encephalitis The infection}

Acanthamoeba granulomatous encephalitis is a rare infection that almost always proves fatal. The cutaneous infections can lasts for months but the involvement of the CNS can result in fatal consequences within days. The routes of entry include lower respiratory tract followed by haematogenous spread. Skin lesions may provide direct entry into the bloodstream, bypassing the lower respiratory tract. Amoebae entry into the CNS most likely occurs at the sites of the blood-brain barrier. In addition, olfactory neuroepithelium provides another route of entry into the CNS and has been used in the experimental models (27). The majority of AGE infections are limited to individuals with weakimmune system (i.e., immunocompromised patients). In addition, patients suffering from other infections such as diabetes, malignancies, malnutrition, alcoholism, or have debilitated immune systems due to immunosuppressive therapy or other complications may also contract AGE infections. The AGE has become a major concern in human health. This is due to increasing numbers of human immunodeficiency virus infection (HIV) / acquired immunodeficiency syndrome (AIDS) patients. At present, the estimated worldwide number of HIV/AIDS patients is a massive 40 - 45 million (as of 2005), which may be potential AGE susceptible hosts. This number continues to rise sharply (approx. 5 million new reported cases of HIV/AIDS in 2003 alone, while 3 million died of HIV/AIDS). Although highly active antiretroviral therapy (HAART) is effective against opportunistic pathogens such as Acanthamoeba (28), it is not available in many developing countries, particularly Africa. The fact that AGE cases are not being reported in developing countries (especially in Africa) is due to the i) lack of expertise, ii) unreported cases and iii) the lack of proper health care systems.

The clinical symptoms are similar to other central nervous system infections due to microbial pathogens including, headache, fever, behavioral changes, hemiparesis, lethargy, stiff neck, aphasia, ataxia, vomiting, nausea, cranial nerve palsies, increased intracranial pressure, seizures and finally leading to death. This is due to hemorrhagic necrotizing lesions with severe meningeal irritation and encephalitis $(27,29)$. Patients with respiratory infections, skin ulcerations or brain abscesses should be strongly suspected for AGE.

\section{Host defenses}

AGE is a rare disease that usually occurs in immunocompromised or debilitated patients due to HIV infection, diabetes, immunosuppressive therapy, malignancies, malnutrition, and alcoholism or as a secondary infection. This is due to the inability of
Acanthamoeba to evade immune system of immunocompetent individuals. Since the haematogenous spread is a pre-requisite in $\mathrm{AGE}$ infection, complement provides the first line defense against AGE. Complement activation is achieved by i) classical pathway (activated by specific antibodies attached to Acanthamoeba surface); ii) alternative pathways (activated by opsonisation), or ii) mannosebinding lectin pathways (activated by surface composition of Acanthamoeba). The ultimate effects are i) to induce the deposition of complement proteins leading to the formation of the membrane attack complex (MAC) or ii) amoebae opsonisation, followed by their uptake by phagocytes (macrophages, neutrophils).

In support of MAC attack, previous studies have shown that normal human serum exhibits complement-mediated lysis in Acanthamoeba, resulting in up to $100 \%$ of amoebae death $(30,31)$. This is due to the fact that the plasma membranes of Acanthamoeba lack sialic acid (32) or any protective coat or capsule (33) and thus Acanthamoeba are exposed to complement-mediated killing (30). However recent studies have shown that a subpopulation of the virulent strains of Acanthamoeba are resistant to complement-mediated lysis $(34,35)$. But complement pathway in the presence of phagocytes (macrophages/neutrophils) is highly effective in clearing Acanthamoeba (36). Overall, complement pathways and the antibodies together with neutrophils and macrophages show potent amoebalytic activities thus suppressing the infection by clearing amoebae $(35,37)$ (Fig. 4).

Tissues, e.g., CNS

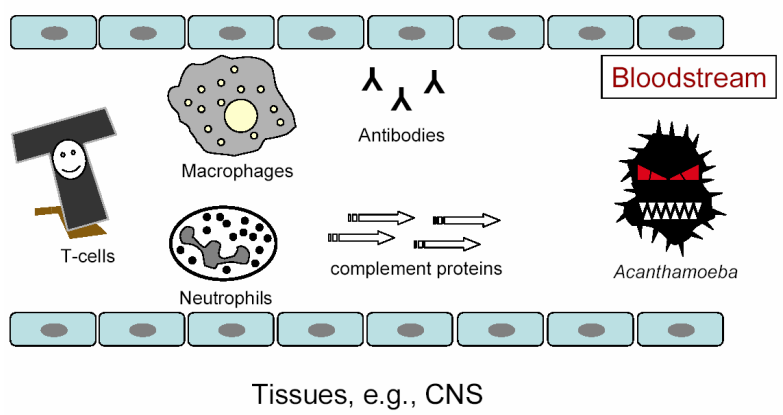

Fig. 4: The immune mechanisms effective against Acanthamoeba in the bloodstream.

It is important to indicate that although macrophages/neutrophils from naïve animals are able to destroy Acanthamoeba, but cells from immune animals exhibit significantly increased amoebalytic activities. The macrophage-mediated killings is 
contact-dependent and is inhibited with cytochalasin D, i.e., an actin polymerization inhibitor (38). This is further confirmed with the findings that conditioned medium obtained from macrophages after treatment with lipopolysaccharide and interferon-gamma had no effects on Acanthamoeba (37). Similarly, the neutrophils exhibit potent amoebacidal effects. The neutrophils-mediated killing is significantly increased in the presence of anti-acanthamoebic antibodies (36). These interactions also stimulate secretion of pro-inflammatory cytokines including IL-1 $\beta$, IL-6 and TNF- $\alpha$ (35,37). Other studies in mice have shown significant increased natural killer cells (NK) activities in Acanthamoeba-infected animals suggesting that NK may also play a role in protective immunity (39). The fact that up to $100 \%$ individuals possess anti-Acanthamoeba antibodies suggest that immunocompetent individuals exhibit Acanthamoeba-specific T-cell responses (in particular CD4-positive T-cells) $(1,40)$. Based on these findings, it is clear that debilitated immune status of the host is a pre-requisite in AGE but the core basis of host susceptibility in contracting AGE are not fully understood and may also involve the host ethnic origin (i.e., genetic basis of the host) or the inability of host to induce specific immune response against these pathogens. Of interest, in a study by Chappell et al., (41), it is shown that Hispanic subjects are 14.5 times less likely to be sero-positive against pathogenic Acanthamoeba than Caucasians. But whether Hispanics may be more susceptible to AGE infection remains to be determined. Future studies will identify the precise host factors, which play an important role in controlling this fatal infection and may help develop therapeutic interventions in the susceptible hosts.

\section{CONCLUSION}

Despite the ubiquitous presence of these organisms in diverse environments, the numbers of infections due to Acanthamoeba have remained low. This is due to the fact that Acanthamoeba are opportunistic pathogens and their ability to produce diseases is dependent on i) host susceptibility, i.e., immunocompromised patients or contact-lens wearers, ii) environmental conditions, i.e., personal hygiene, exposure to contaminated water/soil, and iii) virulence of Acanthamoeba. In normal circumstances, our highly professional immune system is sufficient to control these pathogens. The fact that AGE is limited to immunocompromised patients clearly suggests that normal immune system plays an important role in controlling and/or eradicating these parasites. But due to the complexity of the immune system, the precise factors which contribute to host susceptibility and the associated mechanisms remain unclear. In contrast to

AGE, Acanthamoeba keratitis can occur in normal individuals. But oral immunization can prevent this infection, again suggesting that immune response play important roles. However, the increasing numbers of HIV/AIDS patients, increasing numbers of contact lens wearers and warmer climate will add to the increasing burden of Acanthamoeba infections. Thus there is clear and urgent need for a complete understanding of the pathogenesis and pathophysiology of Acanthamoeba infections, which is crucial for the rationale development of therapeutic interventions and/or design of preventative measures.

\section{ACKNOWLEDGEMENTS}

This work is supported by grants from the Faculty Research Fund, University of London, The Nuffield Foundation and The Royal Society.

\section{REFERENCES}

1. Cursons, RT., T. J. Brown, E. A. Keys, K. M. Moriarty and D. Till, 1980. Immunity to pathogenic free-living amoebae: role of humoral antibody. Infect. Immun., 29(2):401-407.

2. Castellani, A., 1930. An amoeba found in culture of yeast: preliminary note. J. Trop. Med. Hyg., p.160.

3. Volkonsky, M., 1931. Hartmanella castellanii Douglas, et classification des hartmannelles. Arch. Zool. Exp. Gen., 72:317-339.

4. Allen, PG and E. A. Dawidowicz, 1990. Phagocytosis in Acanthamoeba: 1. A mannose receptor is responsible for the binding and phagocytosis of yeast. J. Cell. Physiol., 145:508-513.

5. Alsam, S., J. Sissons, R. Dudley and N. A. Khan, 2005. Mechanisms associated with Acanthamoeba castellanii phagocytosis. Parasitol. Res., 96(6):402-409.

6. Bowers, B and T. E. Olszewski, 1972. Pinocytosis in Acanthamoeba castellanii, kinetics and morphology. J. Cell. Biol., 53(3):681-694.

7. Khan, NA., 2003. Pathogenesis of Acanthamoeba infections. Microb. Pathogen., 34(6):277-285. 
8. Marciano-Cabral, F and G. Cabral, 2003. Acanthamoeba spp. as agents of disease in humans. Clin. Microbiol. Rev., 16(2):273307.

9. Shuster, FL and G. S. Visvesvara, 2004. Free-living amoebae as opportunistic and non-opportunistic pathogens of humans and animals. Int. J. Parasitol., 34(9):1-27.

10. Jager, BV and W. P. Stamm, 1972. Brain abscesses caused by free-living amoeba probably of the genus Hartmannella in a patient with Hodgkin's disease. Lancet, ii:1343-1345.

11. Nagington, J., P. G. Watson, T. J. Playfair, J. McGill, B. R. Jones and A. D. Steele, 1974. Amoebic infections of the eye. Lancet, ii: $1537-1540$.

12. Chang, PC and H. K. Soong, 1991. Acanthamoeba keratitis in non-contact lens wearers. Arch. Ophthalmol., 109(4):463464.

13. Sharma, S., M. Srinivasan and C. George, 1990. Acanthamoeba keratitis in non-contact lens wearers. Arch. Ophthalmol., 108(5):676-678.

14. Jaison, PL., Z. Cao and N. Panjwani, 1998. Binding of Acanthamoeba to [corrected] mannose-glycoproteins of corneal epithelium: effect of injury. Curr Eye Res., 17(8):770-776.

15. Dudley, R., A. Matin, S. Alsam, J. Sissons, A. H. Mahsood and N. A. Khan, 2005. Acanthamoeba isolates belonging to T1, T2, T3, T4 but not T7 encyst in response to increased osmolarity and cysts do not bind to human corneal epithelial cells. Acta Tropica, 95:100-108.

16. Niederkorn, JY., H. Alizadeh, H. Leher and J. P. McCulley, 1999. The pathogenesis of Acanthamoeba keratitis. Microbes Infect., 1:437-443.

17. Nassif, K. F., 1996. Ocular surface defense mechanisms. Infections of the eye. Little, Brown and Company, New York, NY. USA.

18. Qu, XD and R. L. Leher, 1998. Secretory Phospholipase A2 is the principal bactericide for Staphylococci and other Gram positive bacteria in human tears. Infect. Immun., 6(66):2791-2797.

19. Alizadeh, H., S. Apte, M. S. El-Agha, L. Li, M. Hurt, K. Howard, H. D. Cavanagh, J. P. McCulley and J. Y. Niederkorn, 2001. Tear $\operatorname{IgA}$ and serum $\operatorname{IgG}$ antibodies against Acanthamoeba in patients with
Acanthamoeba keratitis. Cornea, 20(6):622627.

20. Leher, H., F. Zaragoza, S. Taherzadeh, H. Alizadeh and J. Y. Niederkorn, 1999. Monoclonal IgA antibodies protect against Acanthamoeba keratitis. Exp. Eye Res., 69(1):75-84.

21. Walochnik, J., A. Obwaller, E. M. HallerSchober and H. Aspock, 2001. AntiAcanthamoeba IgG, IgM, and IgA immunoreactivities in correlation to strain pathogenicity. Parasitol. Res., 87(8):651658.

22. van Klink, F., W. M. Taylor, H. Alizadeh, M. J. Jager, N. van Rooijen and J. Y. Niederkorn, 1996. The role of macrophages in Acanthamoeba keratitis. Invest. Ophthalmol. Vis. Sci., 37(7):1271-1281.

23. Thakur, A and D. P. Willcox, 2000. Contact lens wear alters the production of certain inflammatory mediators in tears. Exp. Eye Res., 70:255-259.

24. Weisman, B and B. Mondino, 2002. Risk factors for contact lens associated microbial keratitis. Cont. Lens Ant. Eye, 25:3-9.

25. Hurt, M, S. Apte, H. Leher, K. Howard, J. Niederkorn and H. Alizadeh, 2001. Exacerbation of Acanthamoeba keratitis in animals treated with anti-macrophage inflammatory protein 2 or antineutrophil antibodies. Infect. Immun., 69(5):29882995.

26. Hurt, M., V. Proy, J. Y. Niederkorn and H. Alizadeh, 2003. The interaction of Acanthamoeba castellanii cysts with macrophages and neutrophils. J. Parasitol., 89(3):565-572.

27. Martinez, AJ., 1991. Infections of the central nervous system due to Acanthamoeba. Rev. Infect. Dis., 13:S399-S402.

28. Pozio, E and M. A. Morales, 2005. The impact of HIV-protease inhibitors on opportunistic parasites. Trends Parasitol., 21(2):58-63.

29. Martinez, AJ., 1985. Free-living Amebas: Natural History, Prevention, Diagnosis, Pathology and treatment of disease. CRC Press, Boca Raton, Florida. p.156.

30. Ferrante, A and B. Rowan-Kelly, 1983. Activation of the alternative pathway of complement by Acanthamoeba culbertsoni. Clin. Exp. Immunol., 54(2):477-485. 
31. Ferrante, A., 1991. Immunity to Acanthamoeba. Rev. Infect. Dis., 13:S403S409.

32. Korn, ED and T. Olivecrona, 1971. Composition of an amoeba plasma membrane. Biochem. Biophys. Res. Commun., 45(1):90-97.

33. Bowers, B and E. D. Korn, 1968. The fine structure of Acanthamoeba castellanii. I. The trophozoite. J. Cell Biol., 39(1):95-111.

34. Sissons, J., A. O. Rivas, J. L. Morales, S. Alsam and N. A. Khan, 2005. Effects of human serum on the virulence properties of Acanthamoeba. XIth International Meeting on the Biology and Pathogenicity of FreeLiving Amoebae, České Budějovice, Czech Republic.

35. Toney, DM and F. Marciano-Cabral, 1998. Resistance of Acanthamoeba species to complement lysis. J. Parasitol., 84(2):338344.

36. Stewart, GL., K. K. Shupe, I. Kim, R. E. Silvany, H. Alizadeh, J. P. McCulley and J. Y. Niederkorn, 1994. Antibody-dependent neutrophil-mediated killing of Acanthamoeba castellanii. Int. J. Parasitol., 24(5):739-42.
37. Marciano-Cabral, F and D. M. Toney, 1998. The interaction of Acanthamoeba spp. with activated macrophages and with macrophage cell lines. J. Eukaryot. Microbiol., 45(4):452-458.

38. van Klink, F., H. Leher, M. J. Jager, H. Alizadeh, W. Taylor and J. Y. Niederkorn,

39. 1997. Systemic immune response to Acanthamoeba keratitis in the Chinese hamster. Ocul. Immunol. Inflamm., 5(4):235-244.

40. Kim, KH., C. O. Shin, and K. Im, 1993. Natural killer cell activity in mice infected with free-living amoeba with reference to their pathogenicity. Kor. J. Parasitol., 31(3):239-248.

41. Tanaka, Y., S. Suguri, M. Harada, T. Hayabara, K. Suzumori and N. Ohta, 1994. Acanthamoeba-specific human T-cell clones isolated from healthy individuals. Parasitol. Res., 80(7):549-53.

42. Chappell, CL., J. A. Wright, M. Coletta and A. L. Newsome, 2001. Standardized method of measuring Acanthamoeba antibodies in sera from healthy human subjects. Clin. Diagn. Lab. Immunol., 8(4):724-730.

43. Martinez, AJ and G. S. Visvesvara, 1997. Free-living, amphizoic and opportunistic amebas. Brain Pathol., 7:583-598. 